\title{
Malignant dermatofibroma: clinicopathological, immunohistochemical, and molecular analysis of seven cases
}

\author{
Thomas Mentzel ${ }^{1}$, Thomas Wiesner ${ }^{2}$, Lorenzo Cerroni ${ }^{2}$, Markus Hantschke ${ }^{1}$, \\ Heinz Kutzner $^{1}$, Arno Rütten ${ }^{1}$, Michael Häberle ${ }^{3}$, Michele Bisceglia ${ }^{4}$, Frederic Chibon ${ }^{5}$ and \\ Jean-Michel Coindre ${ }^{5}$ \\ ${ }^{1}$ Dermatopathology Bodensee Friedrichshafen, Friedrichshafen, Germany; ${ }^{2}$ Deparment of Dermatology, \\ Medical University of Graz, Graz, Austria; ${ }^{3}$ Dermatological Practice, Künzelsau, Germany; ${ }^{4}$ IRCCS-CROB, \\ Referral Cancer Center of Basilicata, Rionero in Vulture (PZ), Italy and ${ }^{5}$ Bergonie Institute, InsermU916 and \\ University Victor Segalen Bordeaux, Bordeaux, France
}

\begin{abstract}
Dermatofibroma (cutaneous fibrous histiocytoma) represents a common benign mesenchymal tumor, and numerous morphological variants have been described. Some variants of dermatofibroma are characterized by an increased risk of local recurrences, and there are a few reported metastasizing cases. Unfortunately, an aggressive behavior cannot be predicted reliably by morphology at the moment, and we evaluated the value of array-comparative genomic hybridization (CGH) in this setting. Seven cases of clinically aggressive dermatofibromas were identified, and pathological and molecular features were evaluated. The neoplasms occurred in four female and in three male patients (mean age was 33 years, range 2-65 years), and arose on the shoulder, buttock, temple, lateral neck, thigh, ankle, and cheek. The size of the neoplasms ranged from 1 to $9 \mathrm{~cm}$ (mean: $\mathbf{3} \mathrm{cm}$ ). An infiltration of the subcutis was seen in five cases. Two neoplasms were completely excised, whereas an incomplete or marginal excision was reported in the remaining cases. Local recurrences were seen in six cases (time to the first recurrence ranged from 8 months to 9 years). Metastases were noted between 3 months and 8 years after diagnosis in six patients. Two patients died of disease, and two patients are alive with disease. Histologically, the primary tumors showed features of cellular dermatofibroma (four cases), cellular/ aneurysmal dermatofibroma (one case), atypical/cellular dermatofibroma (one case), and classical dermatofibroma (one case). Mitotic figures ranged from 3 to 25 per 10 high-power fields, and focal necrosis was present in five cases. Interestingly, malignant transformation from cellular dermatofibroma to an obvious spindle cell/ pleomorphic sarcoma was seen in one primary and in one recurrent neoplasm. Five neoplasms showed chromosomal aberrations by array-CGH, suggesting that these changes may represent an additional diagnostic tool in the recognition of cases of dermatofibroma with a metastatic potential.
\end{abstract}

Modern Pathology (2013) 26, 256-267; doi:10.1038/modpathol.2012.157; published online 21 September 2012

Keywords: dermatofibroma; dermis; fibrous histiocytoma; malignant transformation; sarcoma

Dermatofibroma (fibrous histiocytoma) represents a frequent benign mesenchymal neoplasm of the skin, which rarely may also occur in subcutaneous and deep soft tissues. ${ }^{1}$ Over the years, several morphological variants have been described, and their knowledge is important for the differential diagnosis to more aggressive neoplasms. These variants include epithelioid dermatofibroma, palisading

Correspondence: Professor Dr T Mentzel, Dermatopathology Bodensee, Siemensstrasse 6/1, Friedrichshafen D-88048, Germany. E-mail: mentzel@dermpath.de

Received 23 May 2012; revised 17 July 2012; accepted 17 July 2012; published online 21 September 2012 dermatofibroma, atrophic dermatofibroma, ossifying dermatofibroma, dermatofibroma with osteoclastlike giant cells, lipidized 'ankle-type' dermatofibroma, keloidal dermatofibroma, myxoid dermatofibroma, plexiform dermatofibroma, granular cell dermatofibroma, dermatofibroma with prominent myofibroblastic differentiation, dermatofibroma with intracytoplasmic eosinophilic globules, and cholesterotic dermatofibroma. Clinically important, cellular dermatofibroma ${ }^{2}$ aneurysmal dermatofibroma, ${ }^{3}$ and atypical dermatofibroma ${ }^{4}$ as well as dermatofibromas arising on the face ${ }^{5}$ and in subcutaneous and deep soft tissues ${ }^{1}$ have an increased risk for local recurrence (up to $20 \%$ ) in striking contrast to conventional 
dermatofibromas, that only rarely recur, even if incompletely or marginally excised. During the past years a few examples of cellular, aneurysmal, atypical dermatofibromas as well as of dermatofibromas occurring on the face and in deep soft tissues have been reported that metastasized to lymph nodes and the lungs and even caused death of patients in some instances. ${ }^{1,4,6-14}$ Morphologically, the primary and the metastatic neoplasms showed classical features of the mentioned variants of dermatofibroma, and so far no predictive mophological features have been identified to separate classical benign dermatofibroma from rare metastasizing dermatofibroma. In this study, we investigated seven cases of malignant dermatofibroma in comparison with 10 examples of classical dermatofibroma and to 1 obvious pleomorphic dermal sarcoma not otherwise specified. Given the fact that histology alone is not able to predict the metastatic potential of these tumors reliably, we explored the genetic profile to describe the potential chromosomal abnormalities of malignant dermatofibroma and to compare them with those of 10 true benign dermatofibromas and 1 obvious cutaneous sarcoma. Array-based comparative genomic hybridization (aCGH) is currently the best technique to describe the whole genetic profile in term of copy number variations and shows gains and losses of chromosomes or parts of them. Moreover, this technique is well adapted to formalin-fixed, paraffin-embedded tissue, which is the only tissue available in most cases of the rare tumors.

\section{Materials and methods}

The cases were retrieved from the routine files of the Dermatopathology Bodensee, Friedrichshafen, Germany, and from referral files of the authors, and clinical and follow-up information was obtained from the referring pathologists (clinical features are summarized in Table 1). Cases 3 and 4 have been published in detail already. ${ }^{9,10}$ The tissue in all cases was fixed in $4 \%$ buffered formalin, routinely processed, and embedded in paraffin; $2-4 \mu \mathrm{m}$ thick sections were stained with hematoxylin and eosin. In addition, representative sections were stained immunohistochemically by the labeled Streptavidin Biotin technique using commercially available antibodies; antigen retrieval (microwave heating) was used for all antibodies. Stainings for CD34 (clone Qbend10, dilution 1:80, Immunotech, Marseille, France; clone My10, 1:100, BD Biosciences, Heidelberg, Germany, Proteinase K, pH 6.1), alpha-smooth muscle actin (1A4, 1:500, Sigma Chemical, St Louis, MO, USA; 1A4, 1:500, Dako, Hamburg, Germany),

Table 1 Clinicopathological and molecular findings in seven cases of malignant dermatofibroma

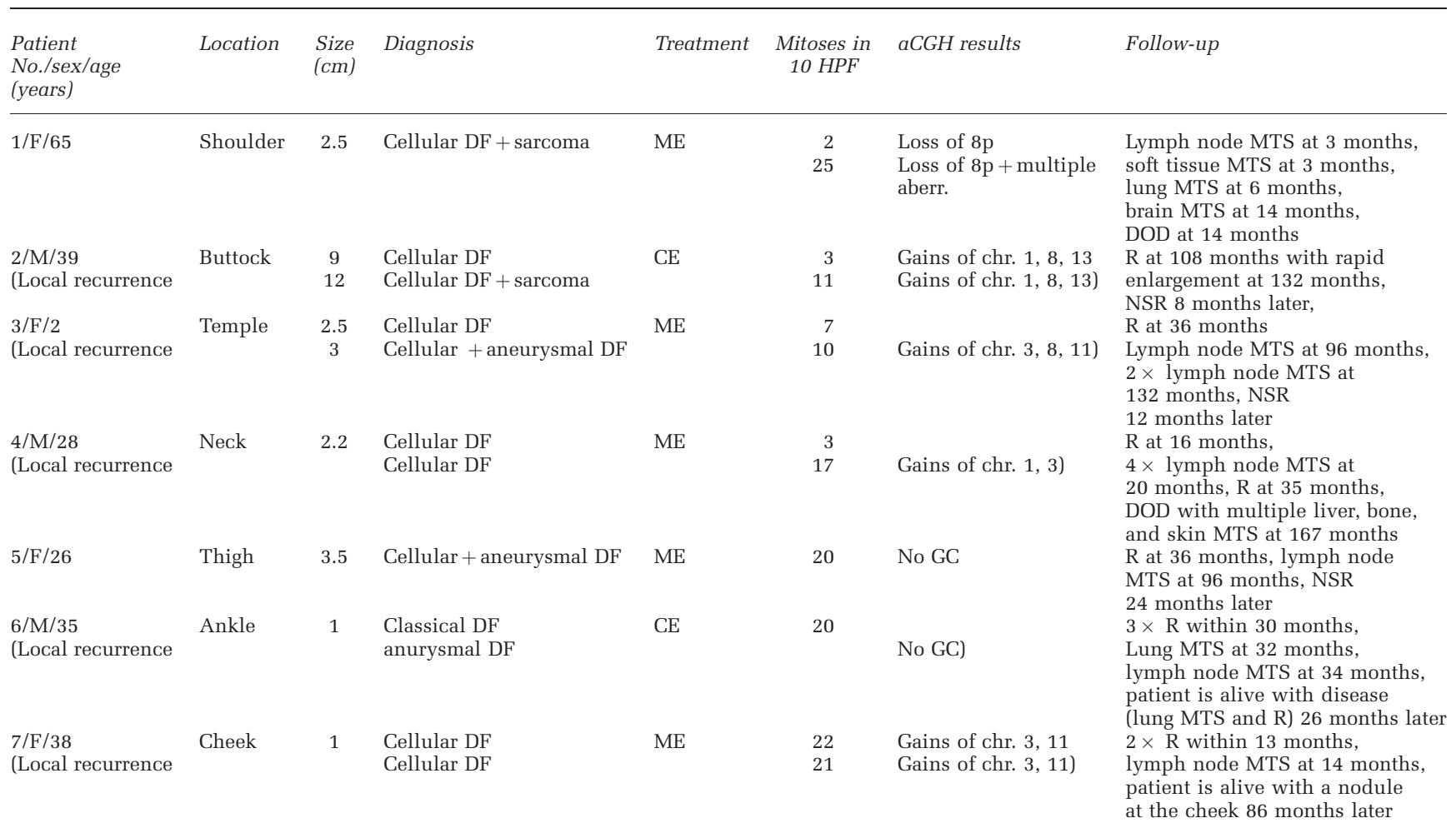

Abbreviations: aberr., aberrations; aCGH, array-comparative genomic hybridization; CE, complete excision; chr., chromosome; DF, dermatofibroma; DOD, died of disease; F, female; M, male; ME, marginal/incomplete excision; MTS, metastasis; no GC, no genetic changes; NSR, no sign of recurrence. 
desmin (D33, 1:100, Dako), S100 protein (polyclonal, 1:2000, Dako), Factor XIIIa (AC-1A1, 1:1000, Thermo Fisher Scientific, Dreieich, Germany), Pancytokeratin (MNF116, 1:500, Dako, Proteinase, pH 9.0), and Ki-67 (SP6, 1:300, Roche Diagnostics, Mannheim, Germany) were available in all cases. Appropriate positive and negative controls were used.

\section{DNA Extraction and Array-Based CGH}

We isolated DNA from formalin-fixed, paraffinembedded tissue to perform a genome-wide, highresolution analysis of chromosomal aberrations as described previously. ${ }^{15}$ Briefly, after DNA isolation with standard methods, $250 \mathrm{ng}$ test and reference DNA (Promega, Madison, WI, USA) were differentially labeled with dCTP-Cy5 and dCTP-Cy3 (GE Healthcare, Piscataway, NJ, USA) using a Bioprime Array CGH Genomic Labeling Kit according to the manufacturer's instructions (Invitrogen, Carlsbad, CA, USA). Genome-wide, aCGH analysis for DNA copy number changes was conducted using an oligonucleotide array containing 60000 probes according to the manufacturer's protocol version 6.0 (Agilent, Santa Clara, CA, USA). Slides were scanned using Agilent's microarray scanner G2505B and analyzed using the Agilent Feature Extraction and DNA Workbench software 6.5.018. ${ }^{16}$

Eight cases of classical dermatofibroma, which had been analyzed previously, and two small typical cases of cellular dermatofibromas were used as negative controls for aCGH and showed no genomic aberrations (data not shown). As positive control for aCGH, we used a dermosubcutaneous metastasis from the chest wall of a 62-year-old male patient, to whom 6 years earlier a 'fibrohistiocytic sarcoma' had been excised on his lateral neck. The metastasis was composed of enlarged, plump spindled tumor cells arranged in confluent formations. The neoplastic cells contained enlarged vesicular nuclei, numerous mitoses (up to 50 mitoses in 10 high-power fields), and did not stain positively for CD34, S-100 protein and alpha-smooth muscle actin. The tumor showed multiple chromosomal aberrations including gains of chromosomes 1, 3, 7, 18, 22 and losses of chromosomes $4,5,6,7,8,9,11,12,13,18,19$ indicating a marked genomic instability and an aggressive biological behavior (data not shown). Within 1 year the patient developed lung and retroperitoneal metastases and died of the disease.

\section{Results}

\section{Case 1}

A 65-year-old female patient complained about a $1.5-\mathrm{cm}$ firm nodule on the right shoulder that slowly enlarged (Figure 1a). A small biopsy was taken and showed a dermal neoplasm composed of spindled and enlarged tumor cells with a pale eosinophilic cytoplasm and enlarged nuclei. Scattered mitotic figures were identified. Immunohistochemically, neoplastic cells stained focally positive for Factor XIIIa and alpha-smooth muscle actin, whereas no expression of CD34, S-100 protein, desmin, and pancytokeratin was seen. The diagnosis of atypical dermatofibroma was made and complete excision was suggested. Two months later a excision was performed, which revealed a $2.5-\mathrm{cm}$ measuring dermal neoplasm showing varying morphological features. Classical morphological features of a cellular dermatofibroma composed of cellular spindle cell fascicles growing around hyalinized collagen bundles without increased proliferative activity and necrosis were noted in one tumor area (Figure 1b). In addition, an abrupt transition to a pleomorphic sarcoma of the dermis and superficial subcutis composed of atypical spindled and pleomorphic tumor cells with enlarged vesicular nuclei was noted. Numerous mitotic figures including atypical mitotic figures were identified in this tumor component (25 mitoses in 10 high-power fields) and areas of tumor necrosis were also present (Figure 1c). The dermatofibroma and sarcoma components were manually microdissected and aCGH was performed separately on both components. The aCGH profile of the dermatofibroma component revealed a loss of the short arm of chromosome 8, but no additional genomic aberrations. The aCGH profile of the sarcoma component showed the same loss on chromosome 8 and in addition multiple other genomic aberrations (Figures 1d and e).

The neoplasm was completely excised, and the minimal distance of neoplastic cells to the basal margin was $1 \mathrm{~mm}$. After 3 months, a $1-\mathrm{cm}$ measuring subcutaneous nodule was identified in the right supraclavicular region and completely excised. Histologically, morphological features of a pleomorphic sarcoma were seen. In addition, a supraclavicular lymph node metastasis was completely excised. The patient received local radiotherapy (66.6 Gy). However, during this treatment a fast growing, partly necrotic tumor developed that showed infiltration of the axillary plexus, and chemotherapy was initiated. After 5 months, the rest of the mainly necrotic neoplasm was completely excised. Unfortunately, pulmonary and cerebral metastases developed after 3 and 11 months, respectively. The pulmonary metastases were removed several times surgically, and the cerebral metastases were treated with radiotherapy. Finally, the patient died from her cerebral metastases.

\section{Case 2}

A 39-year-old male patient presented in 2000 with an ulcerated dermal neoplasm on the left buttock. Grossly, a $9.0 \times 6.5 \times 2.8$-cm measuring skin specimen has been described, and histologically, a cellular spindle cell neoplasm of the dermis with focal infil- 

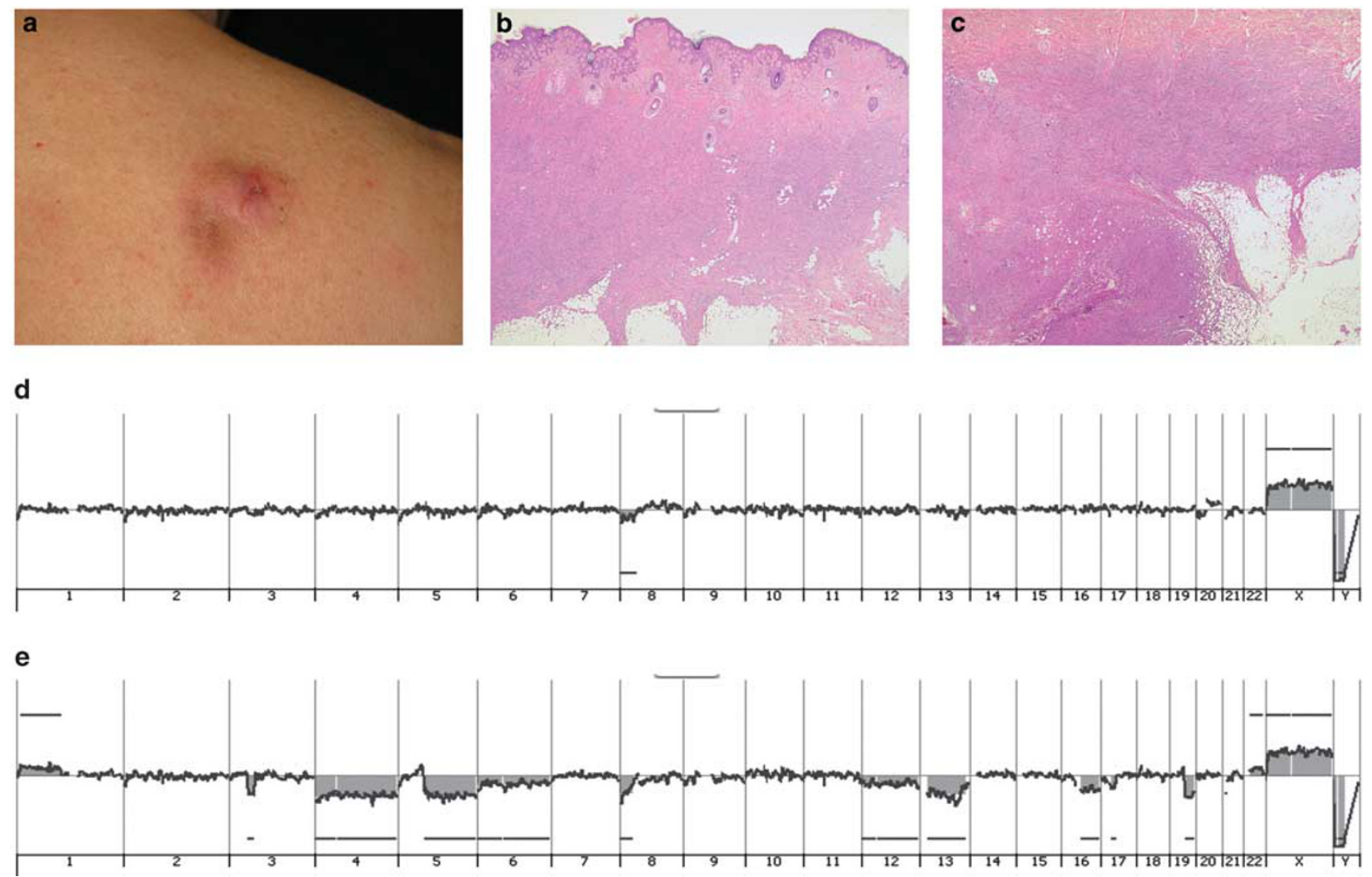

Figure 1 (a) Case 1 arose on the shoulder of a 65-year-old female patient. (b) The excision shows features of cellular dermatofibroma with focal infiltration of the subcutis. (c) A transition from areas of cellular dermatofibroma (right) to an infiltrating, pleomorphic sarcoma is seen. (d) The aCGH profile from the subepidermal, benign component shows a loss of the short arm of chromosome 8, but no additional genomic aberrations. (e) The aCGH profile from the deep lateral, sarcomatous component shows multiple genomic aberration including gains on chromosome 1 and losses on chromosomes $3,4,5,6,8,12,13,16,17$, and 19 . This extremely aberrant aCGH profile is highly suggestive for a highly malignant tumor and resembles the aCGH profiles of a bona fide sarcoma.

tration of the subcutis was reported. The neoplasm was composed of spindled tumor cells with enlarged nuclei and scattered multinucleated giant cells as well as foamy cells were noted. Neoplastic cells were arranged predominantly in fascicles and set in a collagenous stroma (Figure 2a). Scattered mitotic figures (three mitoses in 10 high-power fields) and small areas of tumor necrosis were present. Immunohistochemically, tumor cells stained positively for FXIIIa and focally positive for alpha-smooth muscle actin. The neoplasm was completely excised and the diagnosis of an ulcerated cellular dermatofibroma with no evidence of malignancy was made. The patient reported a slowly growing recurrence in 2009, and a rapid enlargement within 3 weeks was seen in 2011. The local recurrence measured $12 \times 6.5 \mathrm{~cm}$ and was marginally excised. The dermosubcutaneous specimen was covered by an elevated, hyperplastic, and focally ulcerated epidermis, and an exophytic nodule was present (Figure 2b). Histologically, areas consistent with cellular and aneurysmal dermatofibroma were present. In addition, areas composed of atypical spindled tumor cells containing enlarged and hyperchromatic nuclei and scattered multi- nucleated tumor giant cells, which were arranged in fascicles or in cellular sheaths, were present. In these areas, an increased proliferative activity (10-12 mitotic figures in 10 high-power fields) and areas of tumor necrosis were noted (Figures 2c and d). Immunohistochemically, a focal expression of alpha-smooth muscle actin was detected. A wide reexcision was performed and there were no signs of recurrence after 8 months of follow-up. The aCGH profiles of the primary tumor and of the local recurrence showed both chromosomal gains of chromosomes 1, 8, and 13 (Figure 2e). Both profiles were almost identical, suggesting that the local recurrence was caused by the same (or a very similar) neoplastic cell clone. Within the aberrant genomic areas, an oscillating pattern between two copy number states was observed. These frequent oscillations are typical of the recently described chromothripsis phenomenon, which can be seen in at least $2-3 \%$ of all cancers. ${ }^{17}$

\section{Case 3}

A 2-year-old female child presented with a dermal lesion measuring $2.5 \mathrm{~cm}$ in diameter, which occurred 

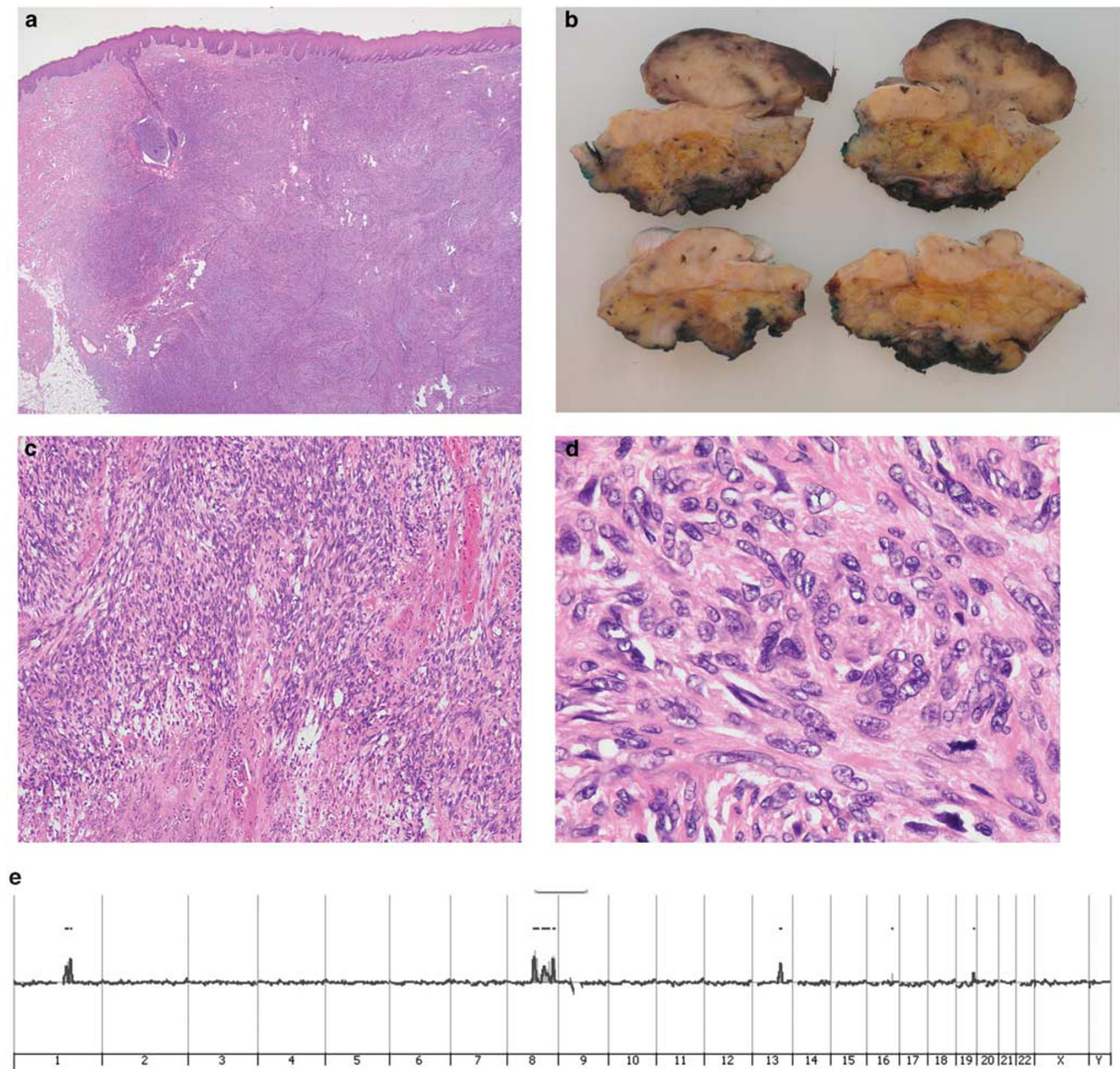

Figure 2 (a) The primary neoplasm of case 2 shows features of cellular dermatofibroma with infiltration of the subcutis. (b) Grossly, the local recurrence of case 2 shows gray-white cut surfaces and an exophytic nodule is present. (c) Higher-power view reveals confluent bundles of atypical spindled cells and areas of tumor necrosis are present. (d) Tumor cells show striking atypia and numerous mitotic figures are present. (e) The aCGH profile of the primary tumor shows chromosomal gains on chromosomes 1,8, and 13 and was almost identically with the aCGH from the local recurrence (data not shown).

on the right temple and was incompletely excised. Histologically, tissue fragments containing a cellular dermatofibroma composed of plump spindled tumor cells showing a fascicular as well as a storiform growth were seen. Numerous mitotic figures (seven mitoses in 10 high-power fields) and small areas of tumor necrosis were identified. Three years later, a local recurrence was completely excised and again showed morphological features of cellular as well as aneurysmal dermatofibroma (Figure 3a). The aCGH profile of the recurrent tumor showed gains in chromosomes 3,8 , and 11
(Figure 3b). At the age of 10 years the patient underwent surgical excision of two right cervical lymph nodes (each $1-2 \mathrm{~cm}$ in size) containing metastatic aneurysmal dermatofibroma. At the age of 13 years another nodular neoplasm measuring $3.5 \mathrm{~cm}$ was detected in the right parotid region and excised. Histologically, the lymphatic tissue was almost completely replaced by pseudocystic and solid tumor areas composed of plump spindled and histiocytoid tumor cells associated with numerous foamy cells, giant cells containing abundant hemosiderin pigment as well as osteoclast-like 

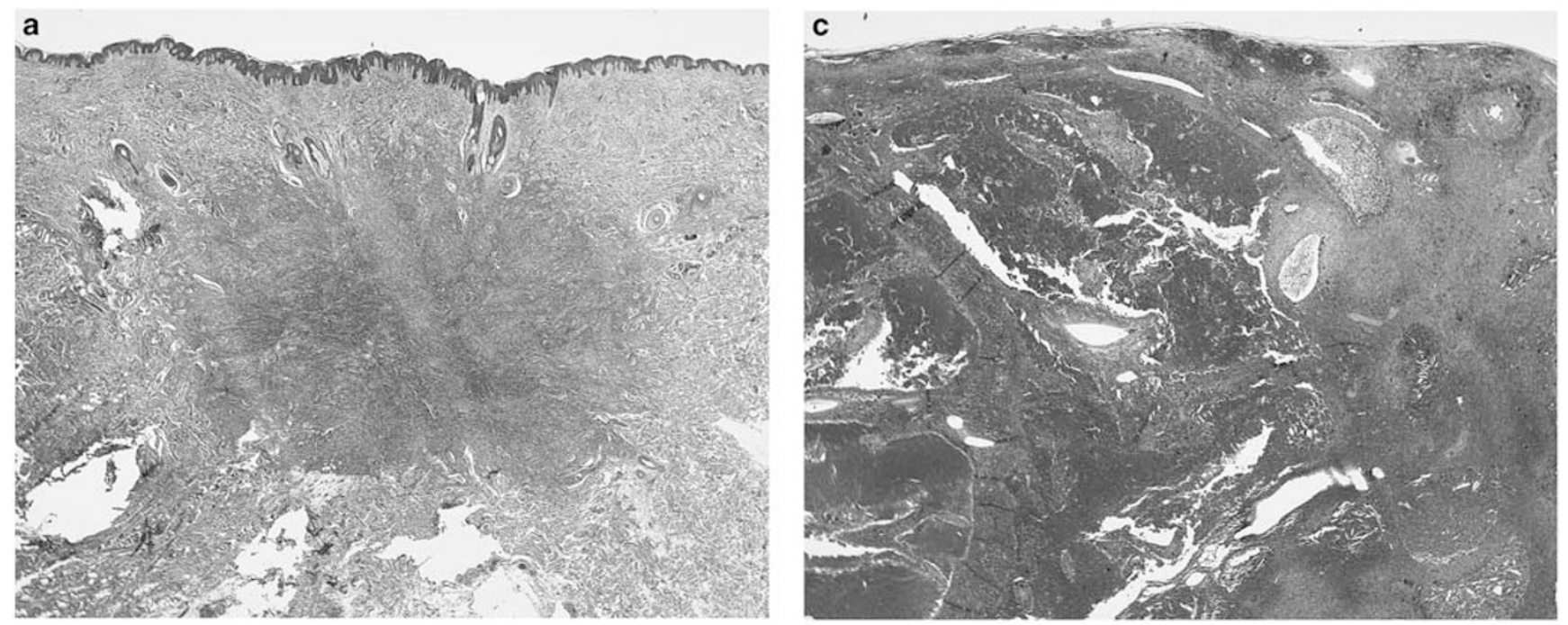

b

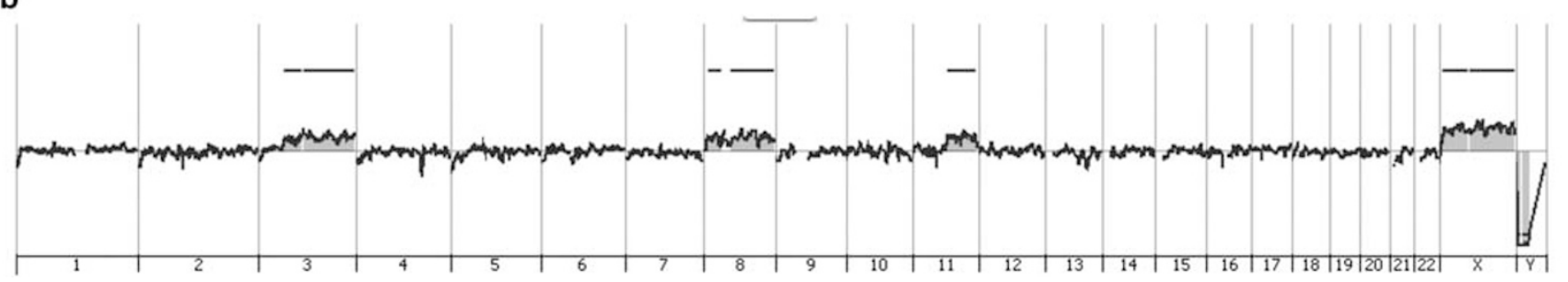

Figure 3 (a) The recurrent neoplasm shows features of cellular dermatofibroma. (b) The aCGH profile reveals gains on chromosomes 3, 8 , and 11. (c) The lymph node metastasis shows features of aneurysmal as well as of cellular dermatofibromas.

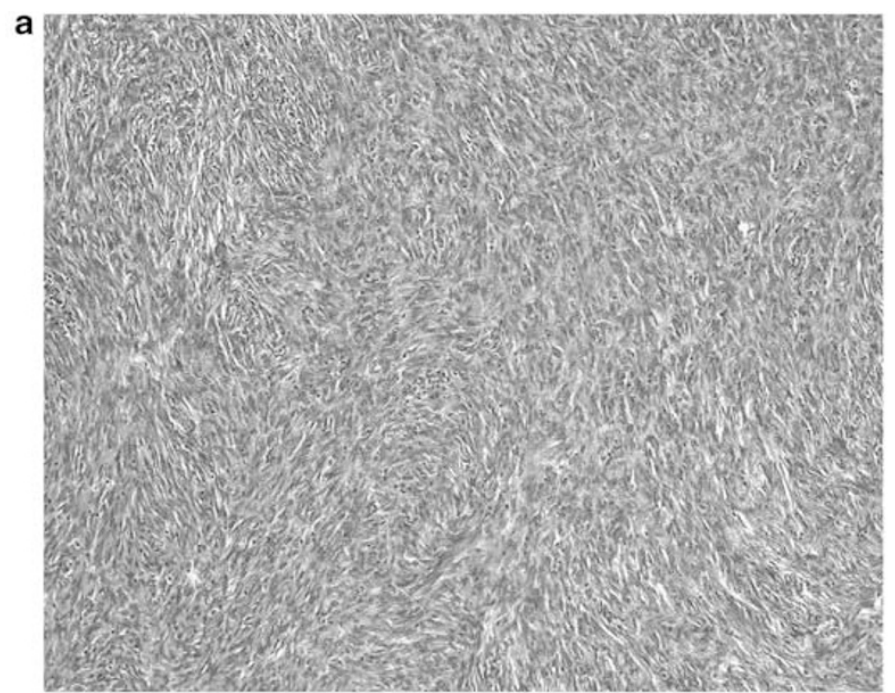

b

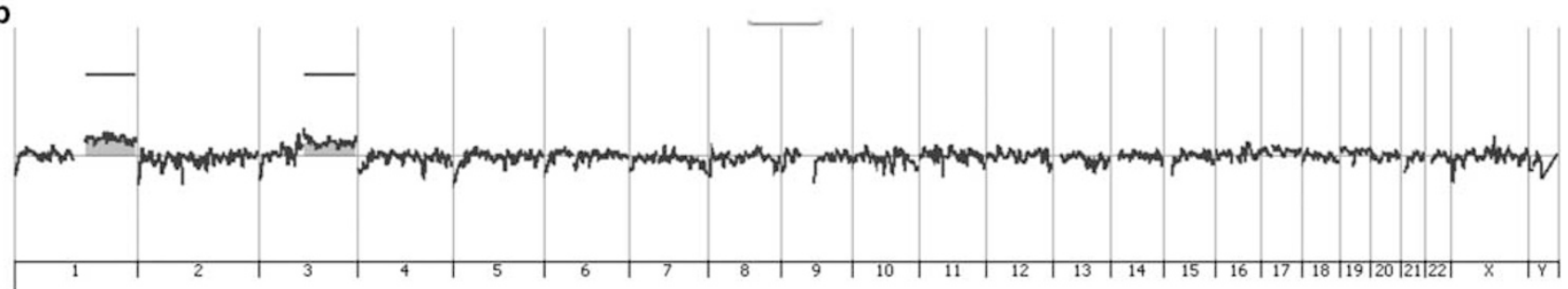

Figure 4 (a) The local recurrence in case 4 reveals a cellular dermal neoplasm composed of spindle-shaped tumor cells with a fascicular and storiform growth. (b) The aCGH profile reveals gains on chromosomes 1 and 3. 
giant cells (Figure 3c), and numerous mitotic figures were detected (up to 15 mitoses in 10 high-power fields). Three months later, right cervical lymph node dissection was performed, and an additional lymph node metastasis was found in 1 out of 21 lymph nodes examined; 12 months later, at the age of 14 years, the young patient was alive and free of disease.

\section{Case 4}

A 28-year-old male patient developed a dermal lesion measuring $2 \mathrm{~cm}$ on the left site of the neck, which was marginally excised. Histologically, morphological features of cellular dermatofibroma were seen. The neoplasm recurred after 16 months as a $3.5 \times 1.5-\mathrm{cm}$ measuring infiltrative neoplasm with infiltration of the subcutis and underlying skeletal muscle, which was surgically excised and histologically examined. The neoplasm was composed of enlarged plump spindled cells arranged in storiform and fascicular growth patterns. Numerous mitotic figures (17 mitoses in 10 high-power fields) and microscopic foci of necrosis were detected (Figure 4a). Immunohistochemically, a focal expression of alpha-smooth muscle actin was noted, whereas cytokeratins, S-100 protein, CD34, and desmin were negative. The aCGH profile revealed gains of chromosomes 1 and 3 (Figure 4b). Four months later, left cervical lymph node dissection was performed and in 4 out of 18 histologically examined lymph nodes metastatic deposits of cellular dermatofibroma were present. After 15 months, the patient developed a second local recurrence that was widely excised, and 11 years later the patient died from lung cancer with liver, bone, and skin metastases.

\section{Case 5}

A 26-year-old female patient presented with an ulcerated dermal nodule on the left thigh. Grossly, a $3.5 \times 2.5-\mathrm{cm}$ measuring lesion was described and was marginally excised. The cellular dermosubcutaneous neoplasm showed features of cellular and aneurysmal dermatofibroma and was composed of plump spindled and histiocytoid tumor cells arranged in a storiform, as well as fascicular growth pattern. Numerous foamy cells and multinucleated giant cells were seen. The tumor cells contained enlarged but relatively uniform nuclei, however, numerous mitotic figures (up to 20 mitoses in 10 high-power fields) and areas of hemorrhagic necrosis were present (Figures 5a and b). Immunohistochemically, neoplastic cells were negative for alphasmooth muscle actin, desmin, S-100 protein, CD34, and cytokeratins. The aCGH profile did not reveal any chromosomal aberrations indicating that the genetic aberrations were only present in a small percentage of the tumors cells or that the genetic aberrations were not detectable by aCGH (eg, balanced
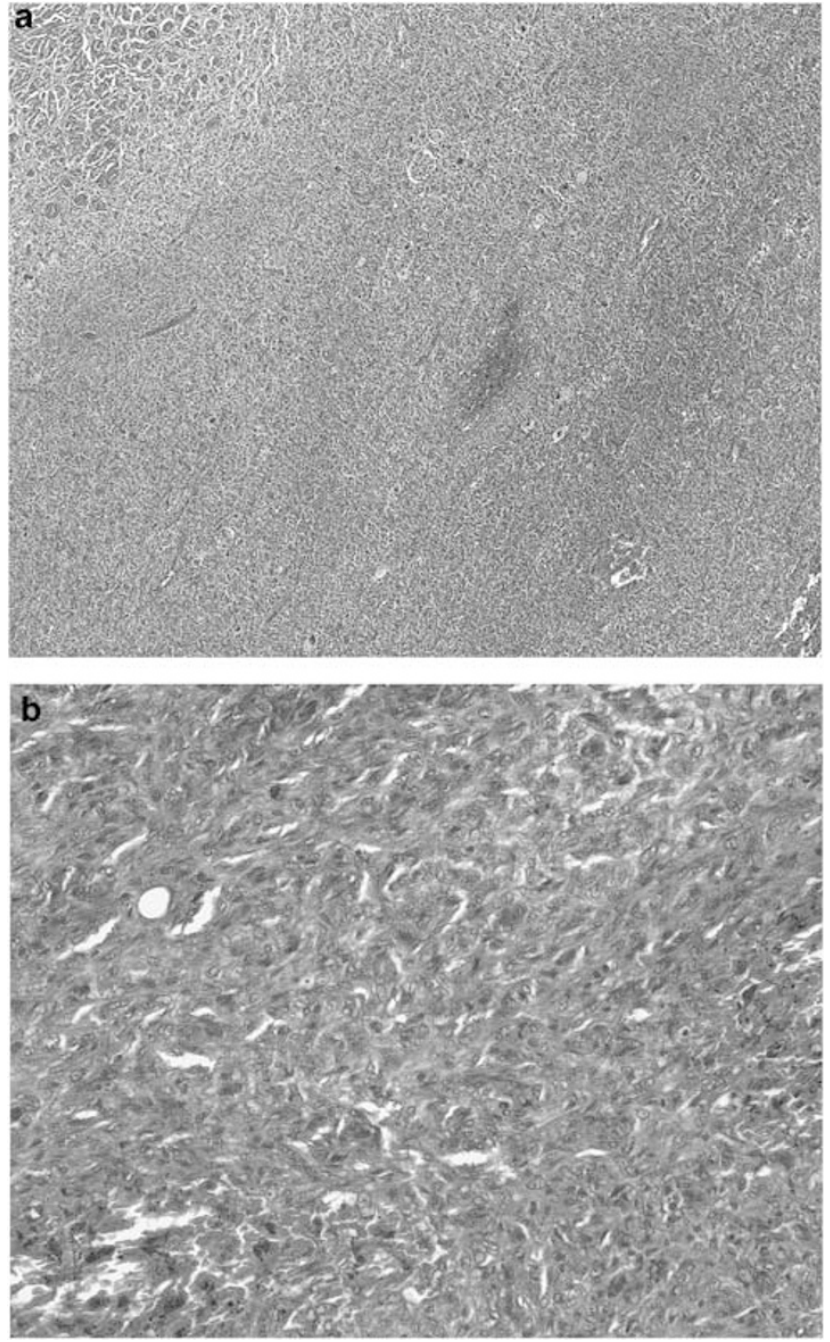

Figure 5 (a) Higher-power view of the primary neoplasm shows features of aneurysmal and cellular dermatofibromas. (b) Tumor cells contain an ill-defined, pale eosinophilic cytoplasm, and slightly enlarged plump spindled nuclei. Numerous mitotic figures are seen.

translocations, point mutations). Three years later a small local recurrence, measuring $1 \mathrm{~cm}$ in diameter, was completely excised. Five years later a lymph node metastasis in the left inguinal region, measuring $6.0 \times 4.0 \times 3.5 \mathrm{~cm}$, was excised and showed morphological features of a partly cystic partly solid dermatofibroma. The patient is alive and has been free of tumor 10 years after surgical excision of the primary tumor.

\section{Case 6}

A 35-year-old male patient developed a firm dermal lesion on the right ankle, measuring $1 \mathrm{~cm}$ in diameter that was completely excised. Low-power view showed a typical example of dermatofibroma without infiltration of the subcutis composed of plump spindled tumor cells, which were arranged 

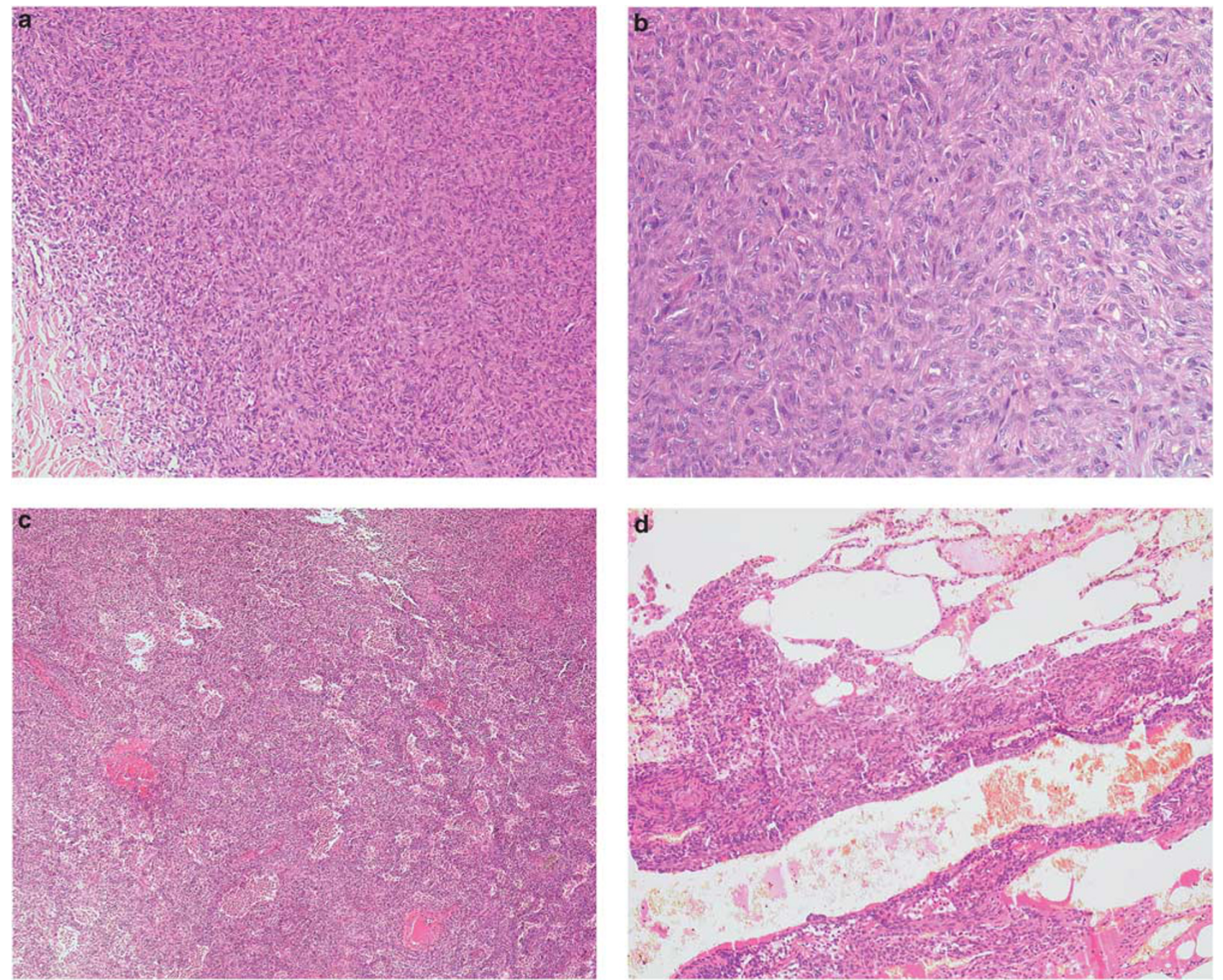

Figure 6 (a) The neoplasm is composed of relatively bland, plump spindled tumor cells arranged in a storiform growth pattern. (b) Despite a bland cytomorphology numerous mitotic figures are present. (c) The local recurrence in case 6 shows features of aneurysmal dermatofibroma. (d) The lung metastasis shows features of aneurysmal dermatofibroma and nests of foamy cells are present.

predominantly in a storiform growth pattern. A lateral extension of tumor cells close to a margin was noted. Despite a bland cytomorphology of neoplastic cells, numerous mitotic figures (up to 20 mitoses in 10 high-power fields) were noted. However, no areas of tumor necrosis were present (Figures 6a and b). Three local recurrences occurred within 30 months. The third local recurrence represented a deep dermal/subcutaneously located cellular neoplasm composed of plump spindled and histiocytoid tumor cells containing slightly enlarged nuclei. However, up to 12 mitotic figures in 10 high-power fields were noted (Figure 6c). aCGH was performed from one of the local recurrences, but no chromosomal aberrations were found. These results indicate that other genetic changes, which are not detectable by aCGH, caused the malignant transformation of this tumor. At 32 months bilateral lung nodules developed and were excised, and histologically, a proliferation of plump spindled and histiocytoid cells including foamy cells was seen (Figure 6d). At 34 months, a lymph node metastasis arising in the right inguinal region was excised, and histologically, morphological features of aneurysmal dermatofibroma were noted. The patient is alive with tumor disease (lung metastases and local recurrence) after 5 years of follow-up.

\section{Case 7}

A 38-year-old female patient developed in August 2003 a firm cutaneous nodule on the cheek measuring $1 \mathrm{~cm}$ in largest diameter that was incompletely excised. Histologically, a cellular, dermal neoplasm with focal infiltration of the subcutis was seen, which was composed of plump spindled tumor cells arranged in storiform and fascicular growth patterns. Tumor cell nuclei were slightly enlarged, and an increased number of mitotic figures (up to 22 

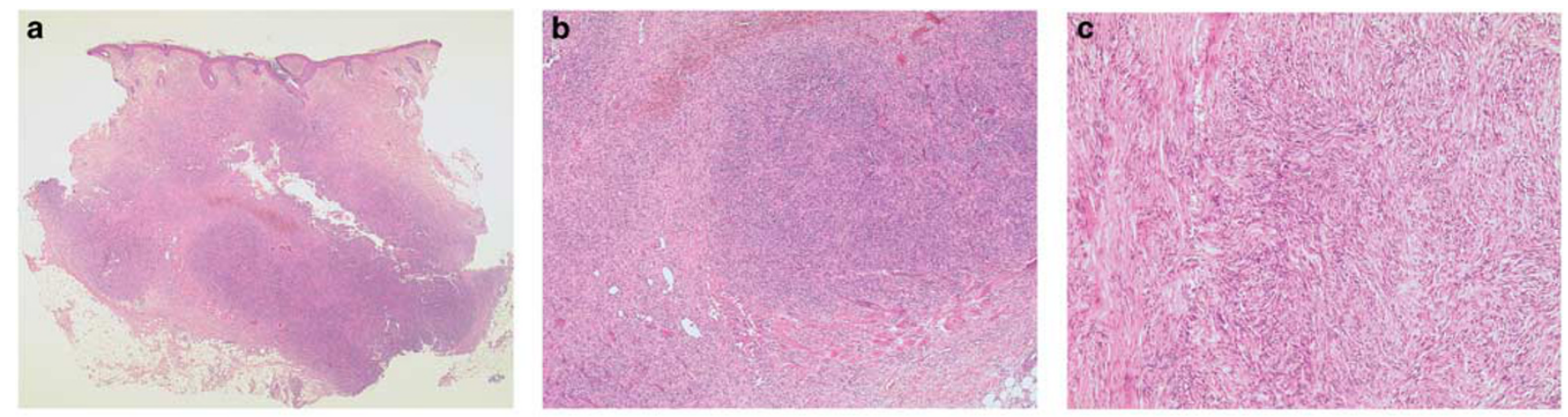

d

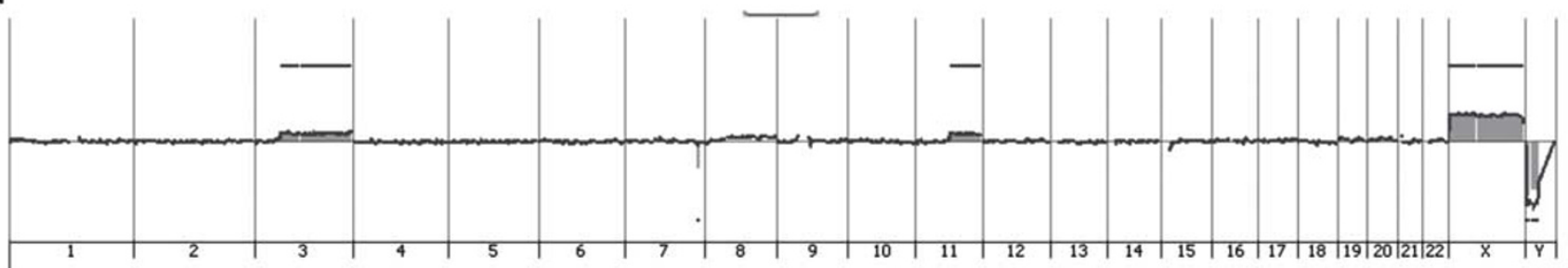

Figure 7 (a) The primary neoplasm in case 7 shows a cellular dermal neoplasm with infiltration of the subcutis. (b) The neoplasm is composed of spindled tumor cells arranged in a fascicular and storiform growth pattern. (c) The local recurrence shows features of classical and cellular dermatofibromas. (d) The aCGH profile of the primary tumor and of the local recurrences are identical and show chromosomal gains on chromosomes 3 and 11 .

mitoses in 10 high-power fields) was noted in the cellular areas; no areas of tumor necrosis were present. The tumor extended to the deep and the lateral margins (Figures $7 \mathrm{a}$ and b). Immunohistochemically, a focal expression of alpha-smooth muscle was noted, the Ki-67 index was scored at $15 \%$. The diagnosis of cellular dermatofibroma occurring on the face was established. At 8 and 13 months, respectively, local recurrences were excised and a satellite nodule was noted and completely excised. The local recurrence showed again morphological features of cellular dermatofibroma and the proliferative activity had not increased in comparison with the primary neoplasm. The satellite nodule showed features of dermal cellular dermatofibroma and was composed mainly of spindle cell fascicles.

Scattered mitotic figures were seen (two to three mitoses in 10 high-power fields), and areas of tumor necrosis were not evident (Figure 7c). The aCGH profiles of the primary tumor and of the local recurrence were identical and showed chromosomal gains of chromosomes 3 and 11 (Figure 7d). At 14 months a cervical lymph node metastasis was completely excised. The patient is alive with a newly recurrent nodule in the cheek at 100 months.

\section{Discussion}

We describe seven cases of unusual dermatofibroma, of which two cases showed morphologically malignant transformation to obvious cutaneous sarcomas (one in the primary and one in the local recurrence), and of which six cases developed lymph node, visceral, and/or soft tissue metastases. Dermatofibroma represents one of the most common mesenchymal lesions of the skin and is characterized by an enormous clinicopathological variation with numerous characteristic and reproducible morphological variants. Although some authors argue that dermatofibroma is a reactive and non-neoplastic condition and represents an inflammatory disease, ${ }^{18}$ the evidence of clonality, ${ }^{19}$ and the fact that some cases of dermatofibroma (especially the cellular, aneurysmal and atypical variants) are potentially recurring lesions that may reach a considerable size and show high cellularity, increased atypia, and proliferative activity support the view that dermatofibroma is indeed a true neoplasm. Even more interesting is the fact that cases of cellular, aneurysmal, and atypical dermatofibroma may very rarely metastasize and cause death of patients and that the development of metastases in a very common benign mesenchymal tumor seems to be unpredictable morphologically. In addition, an obvious malignant transformation in a longstanding dermatofibroma showing rapid enlargement and the presence of a pleomorphic sarcoma developing secondarily in a dermatofibroma has been reported. ${ }^{20}$

Whereas it is well known that about $5 \%$ of patients with von Recklinghausen's disease may develop a malignant peripheral nerve sheath tumor within a plexiform neurofibroma, it was commonly accepted that malignant transformation of benign mesenchymal tumors of the skin and soft tissues does not occur or does so only exceptionally. 
However, during the past years, malignant transformation has been described in some occurrences, eg, in cases of schwannoma, ${ }^{21}$ and of hemangioma/ vascular malformation, ${ }^{22}$ and it has been suggested that there could exist a biological continuum of benign, atypical, and malignant lipogenic neoplasms. ${ }^{23-25}$ Given the fact that premalignancy represents a biological rather than a morphological concept, ${ }^{26}$ it is not surprising that a small group of premalignant mesenchymal neoplasms exist, which may progress to sarcomas with metastatic potential but have not attained all morphological features of malignancy at the time of first presentation.

The cases of metastasizing dermatofibroma reported in the literature and our cases occurred in children and adults, with no preferred anatomic site, and although the majority of metastases were preceded by one or more local recurrences, this was not always the case (Table 2). Similarly, metastases were seen also in cases that had been completely excised. ${ }^{10,11}$ Although the majority of metastasizing cases of dermatofibroma were seen in the cellular, aneurysmal, and atypical variants, case 6 in our seriey represents a relatively small and classical dermatofibroma composed of plump spindled tumor cells, which were arranged predominantly in a storiform growth. Whereas two cases were purely dermal neoplasms, the other five cases showed focal infiltration of the subcutis. Interestingly, all seven cases reported herein were characterized by the presence of numerous mitotic figures and focal areas

Table 2 Review of cases of metastasizing/malignant dermatofibroma $^{\text {a }}$

\begin{tabular}{rlll}
\hline $\begin{array}{l}\text { Case } \\
\text { no. }\end{array}$ & Reference & $\begin{array}{l}\text { Sex/age of } \\
\text { patients (years) }\end{array}$ & Anatomic site \\
\hline 1 & 7 & $\mathrm{M} / 18$ & Thigh \\
2 & 7 & $\mathrm{M} / 33$ & Neck \\
3 & 8 & $\mathrm{M} / 65$ & Unknown \\
4 & 8 & $\mathrm{M} / 30$ & Back \\
5 & 9 & $\mathrm{M} / 27$ & Thigh \\
6 & 9 & $\mathrm{M} / 22$ & Thigh \\
7 & 9 & $\mathrm{M} / 28$ & Neck (present case 4) \\
8 & 6 & $\mathrm{M} / 33$ & Back \\
9 & 4 & $\mathrm{Not}$ stated & Not stated \\
10 & 4 & $\mathrm{Not}$ stated & Not stated \\
11 & 10 & $\mathrm{~F} / 36$ & Knee \\
12 & 10 & $\mathrm{~F} / 2$ & Scalp (present case 3) \\
13 & 1 & $\mathrm{M} / 35$ & Mediastinum \\
14 & 1 & $\mathrm{M} / 51$ & Back \\
15 & 12 & $\mathrm{~F} / 36$ & Shoulder \\
16 & 20 & $\mathrm{~F} / 45$ & Shoulder \\
17 & 14 & $\mathrm{M} / 54$ & Chest wall \\
18 & 14 & $\mathrm{M} / 29$ & Back \\
19 & 13 & $\mathrm{~F} / 29$ & Thigh \\
20 & Present case 1 & $\mathrm{F} / 65$ & Shoulder \\
21 & Present case 2 & $\mathrm{M} / 39$ & Buttock \\
22 & Present case 5 & $\mathrm{F} / 26$ & Thigh \\
23 & Present case 6 & $\mathrm{M} / 35$ & Ankle \\
24 & Present case 7 & $\mathrm{F} / 38$ & Cheek \\
& & & \\
\hline & & &
\end{tabular}

Abbreviations: F, female; $\mathrm{M}$, male.

${ }^{a}$ In addition, a series of 15 cases of metastasizing dermatofibromas have been presented and published as an abstract most recently. ${ }^{11}$ of tumor necrosis were noted in five cases. However, it has been stated that an increase of mitotic activity even with the presence of atypical mitotic figures and areas of tumor necrosis can be seen also in cases of biologically benign dermatofibromas, especially in the cellular and atypical variants. ${ }^{2,4}$ In other words, although a poor clinical outcome was seen most often in large cases with infiltration of the subcutis, which showed an increased proliferative activity and areas of tumor necrosis, it seems that we are unable to predict exactly the clinical outcome of this very common cutaneous neoplasm at the moment by morphology alone, and that a very small group of cases of dermatofibroma exist, which may metastasize and even may cause death of the affected patients. ${ }^{10,11}$

Genomic instability and chromosomal aberrations are a hallmark of human cancers. ${ }^{27}$ Benign tumors, like common dermatofibroma, usually show no chromosomal aberrations, but the vast majority of sarcomas typically display a high level of genomic instability manifested by numerous chromosomal aberrations. It has been shown that cases of so-called malignant fibrous histiocytoma (pleomorphic sarcoma not otherwise specified) show multiple genetic aberrations in most of the cases analyzed, ${ }^{28-30}$ whereas karyotypic alterations in, ie, deep fibrous histiocytoma are sporadic and rare. ${ }^{31}$

In this study, five of seven malignant dermatofibroma samples showed chromosomal aberrations by aCGH. The pattern of chromosomal aberrations included multiple gains and losses of whole chromosomes and chromosomal parts. The excision of case 1 showed two components, areas of classical cellular dermatofibroma and a transition to a pleomorphic sarcoma with increased atypia, mitotic figures, and areas of tumor necrosis. In aCGH, both components showed the same loss on chromosome 8, but the sarcoma component showed additionally numerous other genomic aberrations, suggesting that in rare circumstances dermatofibroma can progress to a sarcoma. The recurrent genomic aberrations were gains on chromosome 3 ( $42 \%$; cases 3, 4, and 7), chromosome 1 (29\%; cases 2 and 4), chromosome 8 (29\%; cases 2 and 3), and chromosome 11 (29\%; cases 3 and 7). Four of seven cases (cases 2, 3, 4, and 7) showed genomic aberrations on two or three chromosomes, which is a higher number than the genomic aberrations found in common dermatofibroma, but considerably less than in histological bona fide sarcoma. The varying number of chromosomal aberrations seen in these lesions may therefore reflect a biological continuum from benign (common dermatofibroma-no chromosomal aberrations) over atypical (atypical dermatofibroma (some chromosomal aberrations) to malignant neoplasms (malignant dermatofibroma/sarcomamany chromosomal aberrations).

Although histology is the best technique to establish the correct diagnosis and to evaluate the prognosis in most cases, it is sometimes limited and 
unable to predict the clinical outcome reliably, as it happens in very rare metastasizing dermatofibromas. Morphology is the result of genetic and epigenetic changes, and the pathologist should keep their importance in mind when reviewing cases. Histology is the best clue for suspicion of genetic lesions and pathologists use this fact in their daily practice for performing genetic analyses on selected cases of mesenchymal tumors, such as sarcomas with a specific translocation, which are composed of small monotonous round or spindle cells, and well differentiated and dedifferentiated liposarcomas for MDM2 amplification. Some tumors can show the same histology but have different prognosis and different genetic lesions, such as dedifferentiated liposarcomas and undifferentiated pleomorphic sarcomas or leiomyosarcomas and rhabdomyosarcomas, and therefore, genetic analysis is very helpful in these situations. On the other hand, clinical context and histology are essential for the whole interpretation, as the same genetic lesion can lead to very different tumors, such as the $t(12 ; 22)$ (EWSR1;ATF1) with clear cell sarcoma and angiomatoid fibrous histiocytoma.

In conclusion, we report seven cases of clinically aggressive dermatofibroma with recurrences and metastases. All cases were characterized morphologically by the presence of mitotic figures and areas of tumor necrosis as well as infiltration of the subcutis in the majority of cases. The presence of chromosomal aberrations by aCGH technique even in the morphologically benign-appearing tumor components may represent an additional diagnostic tool in the recognition of cases of dermatofibroma with a metastatic potential, and we showed that cases of malignant dermatofibroma harbor a genomic profile, which is different from that of true benign dermatofibroma. Given that aCGH may be applied in individual cases of dermatofibroma that show suspicious morphological features (large neoplasms with features of cellular, aenurysmal, and atypical dermatofibroma that infiltrate into the subcutis and show an increased number of mitotic figures and areas of tumor necrosis).

\section{Disclosure/conflict of interest}

The authors declare no conflict of interest.

\section{References}

1 Gleason BC, Fletcher CDM. Deep 'benign' fibrous histiocytoma: clinicopathologic analysis of 69 cases of a rare tumor indicating occasional metastatic potential. Am J Surg Pathol 2008;32:354-362.

2 Calonje E, Mentzel T, Fletcher CDM. Cellular benign fibrous histiocytoma. Clinicopathologic analysis of 74 cases of a distinctive variant of cutaneous fibrous histiocytoma with frequent recurrence. Am J Surg Pathol 1994;18:668-676.
3 Calonje E, Fletcher CDM. Aneurysmal benign fibrous histiocytoma: clinicopathological analysis of 40 cases of a tumour frequently misdiagnosed as a vascular neoplasm. Histopathology 1995;26:323-331.

4 Kaddu S, McMenamin ME, Fletcher CDM. Atypical fibrous histiocytoma of the skin. Clinicopathologic analysis of 59 cases with evidence of infrequent metastasis. Am J Surg Pathol 2002;26:35-46.

5 Mentzel T, Kutzner H, Rütten A, et al. Benign fibrous histiocytoma (dermatofibroma) of the face: clinicopathologic and immunohistochemical study of 34 cases associated with an aggressive clinical course. Am J Dermatopathol 2001;23:419-426.

6 De Hertogh G, Bergmans G, Molderez C, et al. Cutaneous cellular fibrous histiocytoma metastasizing to the lungs. Histopathology 2002;41:85-86.

7 Colome-Grimmer MI, Evans HL. Metastasizing cellular dermatofibroma. A report of two cases. Am J Surg Pathol 1996;20:1361-1367.

8 Colby TV. Metastasizing dermatofibroma. Am J Surg Pathol 1997;21:976.

9 Guillou L, Gebhard S, Salmeron M, et al. Metastasizing fibrous histiocytoma of the skin: a clinicopathologic and immunohistochemical analysis of three cases. Mod Pathol 2000;13:654-660.

10 Bisceglia M, Attino V, Bacchi CE. Metastasizing 'benign' fibrous histiocytoma of the skin: a report of two additional cases and review of the literature. Adv Anat Pathol 2006;13:89-96.

11 Doyle LA, Fletcher CDM. Metastasizing 'benign' fibrous histiocytoma: a clinicopathologic study of 15 cases. Mod Pathol 2012;25:119A.

$12 \mathrm{Gu}$ M, Sohn K, Kim D, et al. Metastasizing dermatofibroma in lung. Ann Diagn Pathol 2007;11:64-67.

13 Szumera-Cieckiewicz A, Ptaszynski K. Benign fibrous histiocytoma of the skin metastasizing to the inguinal lymph node. Pol J Pathol 2011;62:183-186.

14 Osborn M, Mandys V, Beddow E, et al. Cystic fibrohistiocytic tumours presenting in the lungs: primary or metastatic disease. Histopathology 2003;43:556-562.

15 Kutzner H, Metzler G, Argenyi Z, et al. Histological and genetic evidence for a variant of superficial spreading melanoma composed predominantly of large nests. Mod Pathol 25:838-845.

16 Wiesner T, Obenauf AC, Cota C, et al. Alterations of the cell-cycle inhibitors p27(KIP1) and p16(INK4a) are frequent in blastic plasmacytoid dendritic cell neoplasms. J Invest Dermatol 2010;130:1152-1157.

17 Stephens PJ, Greenman CD, Fu B, et al. Massive genomic rearrangement acquired in a single catastrophic event during cancer development. Cell 2011;144:27-40.

18 Zelger BG, Zelger B. Correspondence Re: Guillou L, Gebhard S, Salmeron M, Coindre JM. Metastasizing fibrous histiocytoma of the skin: a clinicopathologic and immunohistochemical analysis of three cases. Mod Pathol 2001;14:534-536.

19 Vanni R, Fletcher CDM, Sciot R, et al. Cytogenetic evidence of clonality in cutaneous benign fibrous histiocytoma: a report from the CHAMP Study Group. Histopathology 2000;37:212-217.

20 McCalmont TH, Scheinberg RS. AFX ex BFX. J Cutan Pathol 2011;38:387-390.

21 McMenamin ME, Fletcher CDM. Expanding the spectrum of malignant change in schwannomas: epithelioid malignant change, epithelioid malignant peripheral nerve sheath tumor, and epithelioid angiosarcoma: a study of 17 cases. Am J Surg Pathol 2001;25:13-25. 
22 Rossi S, Fletcher CDM. Angiosarcoma arising in hemangioma/vascular malformation: report of four cases and review of the literature. Am J Surg Pathol 2002;26:1319-1329.

23 DeiTos AP, Doglioni C, Piccinin S, et al. Coordinated expression and amplification of the $M D M 2, C D K 4$, and HMGI-C genes in atypical lipomatous tumours. J Pathol 2000;190:531-536.

24 Mentzel T. Biological continuum of benign, atypical, and malignant mesenchymal neoplasms-does it exist? J Pathol 2000;190:523-525.

25 Mentzel T, Palmedo G, Kuhnen C. Well-differentiated spindle cell liposarcoma ('atypical spindle cell lipomatous tumor') does not belong to the spectrum of atypical lipomatous tumor but has a close relationship to spindle cell lipoma: clinicopathologic, immunohistochemical, and molecular analysis of six cases. Mod Pathol 2010;23:729-736.

26 Seidman JD, Berman JJ. Premalignant nonepithelial lesions: a biological classification. Mod Pathol 1993;6:544-554.
27 Hanahan D, Weinberg RA. Hallmarks of cancer: the next generation. Cell 2011;144:646-674.

28 Larramendy ML, Tarkkanen M, Blomqvist C, et al. Comparative genomic hybridization of malignant histiocytoma reveals a novel prognostic marker. Am J Pathol 1997;151:1153-1161.

29 Chibon F, Mariani O, Mairal A, et al. The use of clustering software for the classification of comparative genomic hybridization data. An analysis of 109 malignant fibrous histiocytomas. Cancer Genet Cytogenet 2003;141:75-78.

30 Weng WH, Wejde J, Ahlen J, et al. Characterization of large chromosome markers in a malignant fibrous histiocytoma by spectral karyotyping, comparative genomic hybridization (CGH9, and array CGH. Cancer Genet Cytogenet 2004;150:27-32.

31 Frau DV, Erdas E, Caria P, et al. Deep fibrous histiocytoma with a clonal karyotypic alteration: molecular cytogenetic characterization of a $\mathrm{t}(16 ; 17)(\mathrm{p} 13.3 ; \mathrm{q} 21.3)$. Cancer Genet Cytogenet 2010;202:17-21. 